\title{
Individuelle und kollektive Verantwortung. Reichweiten und Rechtsfolgen
}

\section{Otto Luchterhandt}

\section{Zusammenfassung}

Das sog. Atomzeitalter hat den Menschen erstmals in eine globale Verantwortung für sein Überleben genommen. Die stürmische Entwicklung von Künstlicher Intelligenz, von maschinellem Lernen und Robotik sowie ihre Leistungsfähigkeit und systemische Vernetzung drohen die Verantwortung des Menschen für sein Handeln auszuhöhlen, sein Schicksal an nicht mehr beherrschbare komplexe technische Prozesse auszuliefern und die humane Welt von innen heraus zu zerstören. Der Beitrag unterstreicht gegenüber solchen Befürchtungen die Grenzen der Künstlichen Intelligenz, die sich aus ihrer maschinellen, funktionalen Eigenart ergeben, referiert die insbesondere von der EU ausgehenden Initiativen, die ethischen und rechtlichen Bedingungen der Verantwortung des Menschen für seine Entscheidungen $\mathrm{zu}$ sichern, und warnt davor, sich einem letztlich rein funktionalen, reduktionistischen Menschenbild anzupassen und zu unterwerfen.

\section{Schlüsselwörter}

Menschsein - Gefährdung durch maschinelles Gehirn-Imitat (Robotik) und funktionellen Reduktionismus · Wahrung der Verantwortung durch Ethik und Recht

O. Luchterhandt $(\bowtie)$

Universität Hamburg, Hamburg, Deutschland

E-Mail: ottolucht@arcor.de

(C) The Author(s) 2021 


\section{Anthropologische Ausgangsüberlegungen}

Auf dem Symposium Zusammenwirken von natürlicher und künstlicher Intelligenz befassen sich Jipp und Steil mit der Frage: „Steuern wir oder werden wir gesteuert?" (Ausarbeitung im vorliegenden Buch). Das Thema weist in Frageform auf die Gefahr hin, dass der Mensch seine Fähigkeit a) zur individuellen Steuerung seines persönlichen Verhaltens und b) zur gemeinschaftlichen Steuerung seiner Gruppe und seiner Umwelt verlieren könnte. In der Qualifizierung des möglichen Steuerungsverlustes als „Gefahr“ steckt bereits eine These und Stellungnahme: wir sollen und wir wollen die Entscheidung über unser - individuelles und kollektives - Schicksal behalten. Wir wollen uns nicht ausliefern an einen von fremder Hand, im Extremfall von einer künstlichen, maschinellen Hand gesteuerten und für uns blind ablaufenden Kausalverlauf, einen Kausalverlauf, dessen Ziel- und Endpunkt wir ebenso wenig kennen wie seine „Zwischenstationen“ und in dem wir nur noch ein auf die Erfüllung von Funktionen reduzierter organischer Faktor sind.

Warum wollen wir uns nicht ausliefern?

Die Antwort auf die Frage liefert der Schlüsselbegriff meines Themas: „Verantwortung“. Die Behandlung, Ausleuchtung und konzeptionelle Entfaltung des Begriffs der Verantwortung vor allem durch Philosophen und Theologen zeigt das mit großer Klarheit. Ich möchte daher zunächst referieren, was einige prominente Autoren in neuerer Zeit dazu geschrieben haben.

\subsection{Der Begriff der Verantwortung im Spiegel von Philosophie und Theologie}

Gegen Ende der 1960er Jahre bemerkte der Schweizer Rechtsphilosoph Hans Ryffel (1967 S. 275-292), dass die Kategorie der Verantwortung bis zu jener Zeit nur vereinzelt Gegenstand grundsätzlicher wissenschaftlicher und monographischer Ergründung gewesen sei (Weischedel 1933). Im Zentrum des Interesses und der Betrachtungen habe die ethische und theologische Frage der individuellen Verantwortung des Menschen für sein Handeln gestanden. In der Tat: Für die Rechtswissenschaftler und Rechtspraktiker standen die Fragen der persönlichen Verantwortlichkeit oder Haftung für rechtswidriges Verhalten in strafrechtlicher und zivilrechtlicher Hinsicht im Vordergrund. Das Problem einer globalen Verantwortung des Menschen für die Menschheit schlechthin spielte keine Rolle. Dieses Problem ist erst nach dem Zweiten Weltkrieg in den Blick 
gekommen, und zwar, wie mir scheint, zunächst aufseiten der protestantischen Kirchen und von Theologen. Der im August 1948 in Amsterdam gegründete Ökumenische Rat der Kirchen (Weltkirchenrat mit Sitz in Genf) bezeichnete in einem seiner Beschlüsse, auf Gegenwart und Zukunft blickend, die verantwortliche Gesellschaft als das Modell der modernen Gesellschaft (Ryffel 1967, S. 275). Dieses Wort, Erkenntnis und Verpflichtung zugleich, muss vor dem Hintergrund von Hiroshima und Nagasaki, d. h. vor dem Eintritt der Menschheit in das sog. Atomzeitalter gelesen werden. Der zu jener Zeit entstehende machtpolitische und weltanschauliche Ost-West-Konflikt, der sich kurz darauf zum Antagonismus zweier atomar hochgerüsteter Militärblöcke verschärfte und die Welt bis zum Ende der 1980er Jahre beherrschen sollte, hat die Menschheit erstmals in ihrer Geschichte mit der realen Möglichkeit ihrer Selbstauslöschung in und durch einen Nuklearkrieg konfrontiert. In seinem 1947 verfassten Theaterstück Die chinesische Mauer lässt der Schriftsteller Max Frisch (1962) „den Heutigen“ sagen ${ }^{1}$ : „Zum ersten Mal in der Geschichte der Menschheit...stehen wir vor der Wahl, ob es die Menschheit geben soll oder nicht. Die Sintflut ist herstellbar. Technisch kein Problem.“ (S. 82 ff.).

Es war, soweit ich es überblicke, Helmut Thielicke, ev. Theologieprofessor an der Universität Hamburg, der mit dem Grundsatzreferat Christliche Verantwortung im Atomzeitalter (gehalten auf dem CDU-Parteitag 1957 in Hamburg) jene völlig neue Lage der Menschheit und die dem Menschen darin zugewachsene politische und persönliche Verantwortung beschrieben und am Tiefsten ausgelotet hat (Thielicke 1957, S. 80-122).

Thielicke hat das Thema, wie es dem bedrängenden Ernst und der Grundsätzlichkeit des Problems entsprach, weit ausholend, behandelt. Er hat einen anthropologischen, theologischen und philosophischen Traktat verfasst und ist darin der inneren Dynamik der Technik, den immanenten Antrieben und Mechanismen von technologischen Entwicklungen auf den Grund gegangen.

Verständlicherweise hat Thielicke sich damals auf das gerade erst voll in das allgemeine Bewusstsein getretene, bedrängendste Problem des Ost-West-Konflikts konzentriert, nämlich die Bewahrung der Menschheit vor der atomaren Selbstzerstörung, also auf die Bedingungen für die Erhaltung des

\footnotetext{
${ }^{1}$ Der Text geht weiter mit: „Je mehr wir (dank der Technik) können, was wir wollen, umso nackter stehen wir da, wo Adam und Eva gestanden haben, vor der Frage nämlich: Was wollen wir? Vor der sittlichen Entscheidung...Entscheiden wir uns aber: Es soll die Menschheit geben! So heißt das: Eure Art, Geschichte zu machen, kommt nicht mehr in Betracht.“(Frisch 1962, S. 82 ff. (141)).
} 
Weltfriedens. Aber Thielicke ist noch darüber hinaus gegangen. Unabhängig von den Gefahren des atomaren Wettrüstens und des atomaren Antagonismus' hat er eine ganz grundsätzliche und deswegen uns anhaltend auch heute beschäftigende Frage in den Blick genommen, nämlich die Frage nach einer möglichen Eigengesetzlichkeit der Technik und der Technologieentwicklung. Es liegt auf der Hand, dass die Möglichkeit und die Reichweite von Verantwortung von der Antwort auf diese Frage entscheidend beeinflusst wird. Denn wenn die Technik immanenten, eigenen Gesetzen folgt, dann wird ihre Beherrschbarkeit und Beherrschung durch den Menschen zu einem erstrangigen Problem. Thielicke kleidet es in die Frage: „Gibt es so etwas wie die Eigengesetzlichkeit der technischen Entwicklung, der Wirtschaft, der Politik wirklich? Und wenn es so etwas geben sollte: Wie kann es dann überhaupt noch eine wirkliche Chance für verantwortliches, und das heißt doch wohl: für ein freies und vom Gewissen inspiriertes Handeln geben?“ (S. 95).

Die von ihm darauf gegebene Antwort scheint mir für das uns hier interessierende Problem der Verantwortung des Menschen in Bezug auf das Zusammenwirken lebender und nichtlebender Entitäten bedeutsam zu sein. Thielicke konzediert mit realistischem Blick, dass die naturwissenschaftlichtechnische Forschung und damit auch die technologische Entwicklung mit einer inneren, gleichsam dialektischen Dynamik abläuft und sich immer von neuem und beschleunigt vorantreibt.

Gleichwohl stellt er fest (S. 102 f., 105), dass die Eigengesetzlichkeit nicht absolut, sondern nur relativ sei. Der Mensch könne in die Prozesse eingreifen, allerdings nicht beliebig, sondern nur unter bestimmten Bedingungen und Voraussetzungen. Gewissen und Verantwortung könnten erstens bei einer Initialentscheidung über die Einführung oder Ingangsetzung technologischer Neuerungen zum Zuge kommen und zweitens an ihren Endpunkten, d. h. bei der realen Nutzung (Thielicke spricht von Konsumierung) der technischen Ergebnisse. Hier liegen die Schlüsselmomente menschlicher Freiheit und damit auch ihres verantwortlichen Gebrauchs.

Es liegt auf der Hand, dass jene Schlüsselmomente sich nicht im Vorhinein bestimmen lassen, sondern je nach Technologie und Situation variieren, und es liegt auch auf der Hand, dass die Initialentscheidungen und Endpunkte technologischer Abläufe Stunden politischer Entscheidungen sind, in welche die ganze Breite verfassungsrechtlicher, sozialer, ökologischer, wirtschaftlicher, kultureller und sonstiger Interessen und Gesichtspunkte einfließen kann und einfließt.

Der bereits erwähnte Rechtsphilosoph Hans Ryffel hat in einem zehn Jahre nach Thielicke verfassten Essay die Eigenart der Verantwortung in der heutigen Welt im Unterschied zur Situation im europäischen Mittelalter untersucht und 
dabei seinen Blick besonders auf die Bedeutung der normativen Ordnungen gerichtet, in welche die Menschen einst hineingestellt waren und jetzt hineingestellt sind (Ryffel 1967, S. 275-292). Ryffel diagnostiziert zwischen ihnen einen prinzipiellen Unterschied. Typisch für die früheren Zustände sei gewesen, dass die normativen Ordnungen mitsamt ihren Anforderungen fraglos verbindlich gewesen seien. Die Pflicht und Verantwortung der Menschen hätten in einem normgemäßen Verhalten bestanden (S. 284 f.). Kleine Gruppen von Theologen und Philosophen hätten autoritativ die geltenden Ordnungen und Normen verwaltet. Die Masse der Menschen sei in sittlicher Hinsicht unmündig gewesen.

Das sei in der heutigen Zeit anders: Absolut gültige, vorgegebene Ordnungen gebe es nicht mehr und könne es heute nicht mehr geben. Alle Ordnungen seien entdogmatisiert worden. Die Verantwortung des Menschen erstrecke sich daher auch auf die normativen Ordnungen als solche.

Verantwortung und Verantwortlichkeit des Menschen seien dadurch aber nicht entfallen. Vielmehr sei die Verantwortung heute ein allgemeines, schlechthin jeden Menschen verpflichtendes sittliches Phänomen. Sie sei im Prinzip grenzenlos geworden. Auch die technischen Zusammenhänge seien in die sittliche Verantwortung einbezogen. „Der Techniker“, so Ryffel, „muß auf das Dasein im Ganzen Bedacht nehmen, wenn es [das Dasein] in einer sittlich verantwortbaren Weise, d. h. menschenwürdig, eingerichtet werden soll. Und wer sich als Nicht-Techniker von der menschenwürdigen, d. h. sittlich verantwortbaren Einrichtung des Daseins in irgendwelchen Bereichen Rechenschaft geben möchte, kommt um den Einbezug der technischen Zusammenhänge nicht herum.“ (S. 291).

Wegen ihrer Relativität vergleicht Ryffel die normativen Ordnungen von heute zwar mit „Spielregeln“, aber er äußert die Überzeugung, dass die Verantwortung als ein sittliches Phänomen auf die Vorstellung und den Bezug auf ein Absolutes nicht verzichten könne (S. 287 f.). Mit dem Maßstab eines menschenwürdigen Daseins deutet er das Absolute an. Dessen Inhalt lässt er zwar offen und spricht eher vorsichtig von einer unverzichtbaren Voraussetzung, aber zwischen den Zeilen lässt Ryffel keinen Zweifel daran, dass das Absolute die seelisch-geistige Substanz ist, die den Menschen zum Menschen macht.

Es blieb dem Altphilologen und Philosophen Georg Picht (1967) vorbehalten, die bisher referierten Aspekte der Verantwortung zusammenzuführen und ihnen weitere Sinngehalte hinzuzufügen. Picht legt die Grundlagen und die Grundstruktur der Verantwortung als christlich-theologische und als philosophische Kategorie sowohl in individueller als auch in kollektiver Hinsicht offen. Grundsätzlicher und radikaler noch als Helmut Thielicke (1957) lenkt er den Blick auf die Universalität der Verantwortung des Menschen im Atomzeitalter. Im Horizont 
der christlichen Lehre vom Jüngsten Gericht sei Verantwortung, geistesgeschichtlich gesehen, eine eschatologische Kategorie. In der heutigen europäischen Welt werde die Universalität der Verantwortung jedoch nicht mehr durch jene Lehre vermittelt, sondern durch das historische Faktum, dass im Atomzeitalter der Menschheit die Verantwortung dafür aufgezwungen werde, ob es eine zukünftige Geschichte der Menschheit geben werde oder nicht. Die menschliche Verantwortung reiche ebenso weit wie die Möglichkeit der Ausübung menschlicher Macht. Durch Wissenschaft und Technik sei deren Reichweite heute universal geworden.

Die Universalität der kollektiven Verantwortung bestimme, so Picht, auch das individuelle Dasein der Menschen und infolgedessen auch das Maß ihrer persönlichen Verantwortung. Im Ergebnis habe sich die Verantwortung in eine Kategorie verwandelt, deren Reichweite über alle Normen der Moral und des Rechts hinausgehe. Sie sei zu einem Oberbegriff aller jener Verpflichtungen geworden, welche die Menschen zwar im Prinzip besäßen, aber aus mannigfachen Gründen nicht oder nicht zuverlässig erfüllen könnten.

Die Verantwortung reiche jedoch noch darüber hinaus, denn wir, so Picht, trügen ,auch die Verantwortung dafür, dass wir die neuen Aufgaben erkennen, für die noch niemand zuständig ist, von deren Lösung aber das Schicksal der Menschen, der Gesellschaft, des Staates und vielleicht sogar das Schicksal der Menschheit abhängen wird" (S. 221 f.).

Abschließen möchte ich den Überblick mit dem Philosophen Hans Jonas (1984) und seiner Monographie Das Prinzip Verantwortung. Versuch einer Ethik für die technologische Zivilisation. Auch Jonas setzt bei der weltgeschichtlich gänzlich neuen Lage der Menschheit an. „Der endgültig entfesselte Prometheus“, so beginnt sein Werk (S. 7), drohe dem Menschen zum Unheil zu werden. Die Verheißungen der modernen Technik seien in eine Bedrohung der Menschheit umgeschlagen. Das ist Jonas (1984) Ausgangsthese. Die zur geschichtlichen Wirklichkeit gewordene Gefahr für die Existenz sowohl der Biosphäre als auch der Menschheit liefern den Ansatz und Schlüssel zu dem ethischen Konzept des Autors: Die manifeste Drohung und Gefahr hätten Fortschrittsglauben und menschheitsbeglückende Utopien früherer Zeiten in begründete Furcht und Bangen um die Zukunft der Menschheit umschlagen lassen. Den ethischen und zugleich politischen Ausweg aus diesem psychologischen Dilemma aber liefere die Verantwortung für ein Handeln, das die Gefahren zu bewältigen geeignet sei. In die Verantwortung als Prinzip sei die Hoffnung notwendigerweise integriert, denn ohne die Hoffnung darauf, dass die Verantwortung positiv zur Wirkung komme, könne das ethische Programm keinen Erfolg haben. 
In gewisser Weise knüpft Hans Jonas Konzept der Verantwortung aus Furcht an Georg Pichts (1967) Gedanken an, dass die Kategorie der Verantwortung auch die Verpflichtung einschließe, mögliche Gefahren für den Menschen und die Menschheit aus einer hemmungslosen Ideologie der technologischen Machbarkeit frühzeitig zu erkennen und ihnen entgegenzutreten.

Es ist offensichtlich: von dem technologischen Durchbruch, der mit dem Phänomen und dem noch zu problematisierenden Begriff der Künstlichen Intelligenz verbunden ist, gehen heute - erneut im globalen Maßstab - völlig neue Gefahren für den Menschen und die Menschheit insgesamt aus. Anders als die permanente und ja noch keineswegs gebannte Bedrohung der Menschheit durch einen Nuklearkrieg, indes nicht weniger grundsätzlich als dieser, macht die technologische Revolution der Künstlichen Intelligenz mit ihren diffusen und daher unabsehbaren Folgen die Frage nach der individuellen und kollektiven Verantwortung des Menschen zu der wohl schwersten und schwierigsten ethischen Last in unserem nun angebrochenen neuen Industriezeitalter.

\subsection{Das hinter dem Begriff der Verantwortung stehende Menschenbild}

Der Begriff und das Wesen von Verantwortung schließt ein bestimmtes Menschenbild ein, nämlich das Bild eines Lebewesens, das Bewusstsein hat, über Autonomie verfügt und Entscheidungsfreiheit besitzt. Am umfassendsten und weitestreichenden wird das, was den Menschen zum Menschen macht, durch den Begriff der Autonomie ausgedrückt.

Was heißt Autonomie? Ich halte mich an die griechischen Wurzeln des Begriffs und bestimme Autonomie mit Selbstgesetzgebung. Der Mensch ist das Wesen, das sich selbst die Maßstäbe, Normen und Vorschriften seines Verhaltens, seines Handelns, Unterlassens und Duldens vorgeben und zur verbindlichen Maxime machen kann. Ich knüpfe dabei an den von Immanuel Kant (1785) formulierten kategorischen Imperativ an: Handle so, dass die Maxime Deines Handelns als allgemeines, d. h. für alle Menschen gültiges Gesetz tauglich ist (Band 6, S. 74-75). Der Mensch ist für sein Verhalten verantwortlich, weil er frei und willensgesteuert handelt und sich sein Handeln mitsamt ihren Wirkungen und Folgen persönlich zurechnen lassen muss ${ }^{2}$.

\footnotetext{
${ }^{2}$ Zur Kritik gegenüber denjenigen Wissenschaftlern, die hartnäckig die Freiheit des Menschen abstreiten, empfehlen sich Höffe (2017) und Strasser (2018).
} 
Hier ist zunächst auf ein philosophisches Grundproblem einzugehen, das sich in der Gegenüberstellung von Mensch und Roboter, von menschlicher und künstlicher Intelligenz stellt. Das ist deswegen erforderlich, weil das Zusammenwirken lebender und nichtlebender Entitäten im digitalen Zeitalter in starkem Maße von dem Phänomen der künstlichen Intelligenz bestimmt und daher auch von Robotik und Robotern geprägt ist.

Man kann das Problem in eine Reihe von Fragen aufgliedern:

Unterscheidet sich Künstliche Intelligenz von menschlicher Intelligenz und, wenn ja, wodurch? Wenn Künstliche Intelligenz sich nach dem heutigen Stand der Technik und Entwicklung noch von der menschlichen Intelligenz unterscheidet, ist das nur ein gradueller, zeitlich befristeter Unterschied, trägt also die Entwicklung von Künstlicher Intelligenz das Potenzial in sich, das Niveau und eine dem menschlichen Gehirn vergleichbare Qualität zu erreichen? Kann, anders formuliert, der Roboter zu einem Wesen mit der Fähigkeit zur Selbstgesetzgebung werden? Ist der mit künstlicher Intelligenz ausgestattete Roboter nicht nur nach dem heutigen Stand der Technik eine Maschine, sondern wird er auch in alle Zukunft eine Maschine sein und bleiben, weil den Roboter vom Menschen, weil die Künstliche Intelligenz von der menschlichen Intelligenz eine unüberbrückbare Kluft trennt, eine Kluft, die auf den allein dem Menschen eigenen Wesensmerkmalen beruht?

Auf diese Fragen werden von den mit der Entwicklung von Künstlicher Intelligenz befassten Praktikern, Ingenieuren, Informatikern und Wissenschaftlern unterschiedliche Antworten gegeben. Zwei Positionen, Erwartungen, Meinungen stehen sich polar gegenüber: erstens die Position des sogenannten Transhumanismus, die erwartet (und daran arbeitet!), dass der Mensch mit der ihm tendenziell überlegenen Künstlichen Intelligenz eine Verbindung eingeht, kraft derer er eine höhere anthropologische Stufe erklimmt ${ }^{3}$.

Dem steht eine Position radikaler Skepsis gegenüber, die den Transhumanismus für eine Utopie, für Science-Fiction hält. Künstliche Intelligenz unterscheide sich als maschinelle Intelligenz wesensmäßig von menschlicher Intelligenz. Die Unterschiede zwischen Mensch und Maschine seien unaufhebbar.

Zwischen diesen beiden Positionen bewegen sich jene Forscher, Ingenieure, Philosophen und sonstige Humanwissenschaftler, die die Frage offen lassen und sich auf die Feststellung beschränken, dass zumindest nach dem heutigen

\footnotetext{
${ }^{3}$ Wikipedia Transhumanismus Artikel (o. J.) - mit weiteren Nachweisen; Günther (2018); Einer der „Propheten“ des Transhumanismus ist Ray Kurzweil. Zu ihm Kreye (2018); ferner Clark (2018); kritisch zur „Maschinenreligion“ auch Klingler (2018).
} 
Stand der Technik und auch in absehbarer Zeit die Entwicklung der Künstlichen Intelligenz noch nicht die Stufe einer universellen Leistungsfähigkeit wie das menschliche Gehirn erreichen werde, sondern nur mehr oder weniger eng und klar definierte Aufgaben werde lösen können ${ }^{4}$.

Meist mischt sich in diese Position allerdings eine grundsätzliche Skepsis hinsichtlich der Möglichkeit der technischen Entwicklung zum Transhumanismus, selbst wenn man sie gutheißen würde. Man kann diese „mittlere Position" als pragmatisch bezeichnen, weil sich ihre Vertreter an das heute in technischer Hinsicht Machbare halten und sich auf die Lösung solcher Probleme konzentrieren (wollen), die den Einsatz von Künstlicher Intelligenz in bestimmten Lebensbereichen - Verkehr, Gesundheitswesen, Verwaltung, industrielle Fertigung usw. - zum Erfolg führen.

Der Streit um die Transhumanismus-Position kann hier offenbleiben. Gleichwohl halte ich es für sinnvoll, einige Bemerkungen zum kategorialen Unterschied zwischen Mensch und Roboter, zwischen Künstlicher Intelligenz und menschlicher Intelligenz zu machen; denn das, was den Menschen zum Menschen macht und ihn wesensmäßig von der Maschine und damit auch vom Roboter unterscheidet, bildet die anthropologische Grundlage für die Fähigkeit des Menschen zur Verantwortung und berechtigt dazu, ihn in die Verantwortung zu nehmen.

Der Mensch ist ein aus der universellen Natur hervorgegangener und in diese Natur eingebetteter Organismus, in dem Leib, Seele und Geist mit einander zu einer untrennbaren Einheit verbunden und verschmolzen sind. Wie jedes natürliche Lebewesen besitzt der Mensch Lebenswillen, ein Geschlecht und Fortpflanzungsfähigkeit; er unterliegt Geburt, Wachstum, Alterung und Tod. Der Mensch hat eine Seele, er hat Gefühle und die Fähigkeit, Gefühle mit anderen Lebewesen zu teilen. Eingebettet in die Familie von Mitmenschen hat der Mensch eine evolutive Geschichte, Sprache, Gedächtnis, komplexes Alltagswissen und Alltagsorientierung sowie ein differenziertes Erinnerungsvermögen ${ }^{5}$. Der Mensch besitzt Intuition (Ingold 2018) und Unterbewusstsein sowie Bewusstsein ${ }^{6}$ und die Fähigkeit zum universellen Denken und Nachdenken über sich selbst, d. h. über

\footnotetext{
${ }^{4}$ Erhellend in Bezug auf die in Fachkreisen vertretenen verschiedenen Positionen die dokumentierte Diskussion in: Schnabel (2018, S. 37-39).

${ }^{5}$ Der Philosoph Reinhard K. Sprenger nennt in bewusst bunter Reihenfolge „Autonomie, Kontextsensibilität, Intuition, Analogiebildung, Gewissen, Sterblichkeit, Sorge, Liebe, Schönheit, Zauber, Frömmigkeit, Neugierde, Unternehmertum, Sympathie, auch Verstehen in einem starken Sinne - alles nicht programmierbar“. Sprenger (2019).

${ }^{6}$ Zum Problem des menschlichen Bewusstseins: Mørch (2018).
} 
alle Teile und Aspekte seiner individuellen Persönlichkeit als körperlich-seelischgeistige Einheit. Die kognitiven Fähigkeiten des Menschen und seines Gehirns speisen sich nicht nur aus der Beherrschung der Sprache und der abstrakten, logischen Verknüpfung von Gedanken, sondern ganz entscheidend auch aus den Impulsen, Eindrücken und Bildern, welche die Sinne laufend dem Gehirn liefern und von ihm verarbeitet werden. Darauf beruht die Fähigkeit des Menschen zur Kreativität, d. h. zur Schaffung von neuartigen Produkten und Objekten oder zur Formulierung neuer Erkenntnisse, gleichsam ex nihilo.

Die für das Wesen des Menschen bestimmende und daher typische Verschmelzung von körperlicher Kreatürlichkeit und geistig-seelischer Bewusstseinssphäre und Reflexion macht den fundamentalen Unterschied zum Roboter und zur künstlichen Intelligenz evident. Der letztlich entscheidende Unterschied ist der, dass künstliche Intelligenz kein Bewusstsein hat (Widmer 2018; Gabriel $2018)^{7}$. Sie erfüllt nur Funktionen, ohne zu wissen, dass sie sie erfüllt, und sie löst nur solche Aufgaben, die der Mensch ihr gestellt hat, und mit dem Material, das der Mensch geliefert hat. Das aber kennzeichnet die Maschine. Sie ist ein Werkzeug, ein Instrument des Menschen, um vom Menschen bestimmte Zwecke zu verfolgen und vom Menschen bestimmte Ergebnisse zu erzielen.

Zweifellos ist es in den letzten Jahrzehnten Ingenieuren und Technikern gelungen, die Leistungsfähigkeit von Künstlicher Intelligenz außerordentlich und insgesamt so weit zu steigern, dass sie für die Lösung immer komplexerer Aufgabenstellungen eingesetzt werden kann. Möglich geworden ist das erstens durch das sogenannte „maschinelle Lernen“ der Träger von Künstlicher Intelligenz und zweitens durch die Entwicklung ,künstlicher neuronaler Netzwerke" (vgl. Armbruster 2018a, b). Vereinfacht und allgemein gesagt beruht beides auf der Nachahmung, auf der Imitation und Modellierung von Prozessen der Informationsverarbeitung im menschlichen Gehirn. Einprägsam hat das der Philosoph Julian Nida-Rümelin (2016) auf den Punkt gebracht: ${ }^{8}$ „Künstliche Intelligenz gibt es im Wortsinne nicht“, schreibt er. „Das, was wir als künstliche Intelligenz bezeichnen, imitiert menschliches Denken zum Teil sehr erfolgreich, wie man schon an jedem Taschenrechner feststellen kann. Computer oder Softwaresysteme oder autonome Fahrzeuge haben aber keine Einstellung zur Welt, sie erkennen nichts, sie entscheiden nichts. Sie simulieren empirische und wertende

\footnotetext{
${ }^{7} \mathrm{Zu}$ dem Problem und der damit verbundenen Kontroverse siehe auch Henn (2018).

${ }^{8} \mathrm{Zu}$ der Problematik aus Sicht eines Politikers siehe Lammert (2018), Kissinger (2018) und aus der Sicht einer Schriftstellerin siehe Hahn (2019).
} 
Einstellungen zur Welt, sie simulieren Erkenntnis und Entscheidung." (Neue Züricher Zeitung, S. 22).

Durch Nachahmung, Imitation und Modellierung von Prozessen der Informationsverarbeitung im menschlichen Gehirn wird nun aber, das ist entscheidend, der Charakter der Künstlichen Intelligenz als ein maschinelles Phänomen nicht verändert (von der Malsburg 2019).

Der Fachausdruck maschinelles Lernen drückt das zutreffend und prägnant aus. Künstliche Intelligenz zeichnet sich zwar durch erstaunliche Rechen- und Rechnerleistungen binnen kürzester Frist aus, also durch Leistungen, die Fähigkeiten des menschlichen Gehirns bei weitem übersteigen, aber sie übertreffen das menschliche Gehirn erstens nur in ausgewählten, überschaubaren und mehr oder weniger begrenzten Bereichen, Aufgabenstellungen und Problemkonstellationen, und zweitens hängt die Leistungsfähigkeit der Künstlichen Intelligenz entscheidend von den Datenmengen ab, mit denen sie vom Menschen gefüttert werden $^{9}$. Damit aber werden technische und qualitative Grenzen von künstlicher Intelligenz sichtbar.

Der maschinelle Charakter von Künstlicher Intelligenz kommt in ihrem Begriff nicht angemessen zum Ausdruck. Der Begriff wird daher zu Recht als unpassend kritisiert (vgl. Günther 2016, S. 23 ff.). Die Bedenken erstrecken sich auf beide Elemente - auf künstlich und auf Intelligenz. Das heißt: treffender wäre es erstens, statt von künstlicher von maschineller Intelligenz zu sprechen, um den kategorialen Unterschied zwischen Maschine (Roboter) und Mensch schon sprachlich bewusst $\mathrm{zu}$ machen. Zweitens sollte man nur mit einem distanzierenden Vorbehalt von Intelligenz sprechen, um den weiten Abstand der maschinellen Intelligenz zum menschlichen Gehirn hervorzuheben. Denn das, was mit dem Begriff der Künstlichen Intelligenz, verbal auf eine anthropologische Höhe gehoben und so gleichsam geadelt wird, stellt, wenn es um den Herstellungsprozess von Robotern geht, in Wahrheit lediglich eine maschinelle Imitation von menschlicher Intelligenz dar, und wenn es um das in einen Roboter einzubauende Ergebnis der Herstellung geht, kann nur von Pseudo-oder Scheinintelligenz, oder, noch präziser und knapper, von einem maschinellen Gehirnimitat gesprochen werden.

\footnotetext{
${ }^{9}$ Auf der Möglichkeit des unbeschränkten Zugangs zu gigantischen Datenmengen beruht in einem nicht zu unterschätzenden Grade die enorme Dynamik, welche die Forschung, Entwicklung und praktische Anwendung der Künstlichen Intelligenz in der Volksrepublik China auszeichnet. Es vergeht kaum eine Woche, in der nicht Medien darüber berichten. Siehe dazu aus jüngster Zeit nur die Reportagen von Müller (2019), Böge (2019), Ankenbrand (2019), und Glaser (2017).
} 
Die Klarstellung dürfte nötig sein, denn, wie Kenner der Materie berichten, besteht eine Tendenz im Milieu der Künstlichen Intelligenz-Forschung und Entwicklung, Roboter zu vermenschlichen (vgl. Lenzen 2018a, b, 2019; Scheer 2019; Betschon 2018a, b; Beschorner 2018), also die prinzipiellen Unterschiede zwischen Mensch und Maschine, zu verwischen. Das kann nicht gut sein, schon deswegen nicht, weil dadurch in der Öffentlichkeit, in der Zivilgesellschaft, in der politischen Publizistik und bei Politikern gefährliche Irrtümer und Fehlvorstellungen gefördert werden, sei es, dass in der Gesellschaft Ängste vor Bedrohungen durch künstliche Intelligenz verstärkt oder dass - umgekehrt naive, völlig überzogene, hoffnungsfroh stimmende Erwartungen an Künstliche Intelligenz geknüpft werden.

Trotz der an dem Begriff der Künstlichen Intelligenz geäußerten fundamentalen Kritik halte ich in dem vorliegenden Rahmen an ihm fest. Das geschieht aus pragmatischen Gründen, weil der aus dem Amerikanischen übernommene Begriff der Künstlichen Intelligenz (artificial intelligence), längst weltweit in den Medien, in der Politik und auch in den Wissenschaften die beherrschende Terminologie zur Beschreibung und Bezeichnung der maschinellen Imitation menschlicher Intelligenz und der Robotik geworden ist und demgemäß auch das Symposium: Zusammenwirken von natürlicher und Künstlicher Intelligenz, prägt.

Der Abstand und kategoriale Unterschied des Roboters mit seiner Künstlichen Intelligenz vom Menschen lässt sich noch an dem Vergleich ihrer Funktionsweise mit dem Tier aufzeigen. Denn im Prinzip nicht anders als das Tier, das instinktgebunden, vom Instinkt gesteuert wird und sich nur in der Bandbreite seines Instinktes ,autonom“ bewegen und „Entscheidungen“ treffen kann, ist der mit maschineller Pseudointelligenz ausgestattete Roboter ex ovo funktionsgebunden. Er kann nur in der Bandbreite und in den Grenzen seiner ihm vom Menschen zugewiesenen und vorgegeben Zwecke und Aufgaben handeln und nur darauf gerichtete Entscheidungen treffen. Die Qualität eines Lebewesens und Geschöpfes gehen dem Roboter ab, denn er ist durch und durch ein vom Menschen gemachtes Instrument und Werkzeug. Der Roboter ist eine Maschine, die einem Menschen gehört, d. h. Eigentum eines Menschen oder eines Unternehmens ist. Er ist nicht Person, sondern Sache im zivilrechtlichen Sinne, und seine Beschädigung oder Zerstörung ist Sachbeschädigung im Sinne des Strafrechts. Während der verstorbene Mensch Gegenstand der Pietät ist und von Mitmenschen zu Grabe getragen wird, ist der zerstörte oder irreparabel funktions(un) fähige Roboter Schrott, „Elektroschrott“, Sondermüll. Er landet auf dem Schrotthaufen, nicht auf dem Friedhof. 


\section{Bereiche des Zusammenwirkens von natürlicher und Künstlicher Intelligenz}

Aus dieser knappen anthropologischen Grundlegung, die ich für eine notwendige Klarstellung halte, ergibt sich die Schlussfolgerung, dass der mit Künstlicher Intelligenz ausgestattete Roboter eben wegen seines maschinellen Charakters immer nur als Instrument und sektoral eingesetzt werden kann, und zwar mit möglichst genau vorgegebenen Zielbestimmungen und Aufgabenbeschreibungen, um sichere, eindeutige, gut verwertbare Ergebnisse zu erzielen, die auch wirtschaftlichen Erfolg verbürgen.

Allerdings hat sich der Einsatz von Künstlicher Intelligenz im gegebenen Rahmen letztlich aus pragmatischen Gründen heute, an der Schwelle zum sogenannten Industriezeitalter $4.0^{10}$, gegenüber den 1990er Jahren außerordentlich erweitert. Robotik und Künstliche Intelligenz haben Eingang gefunden in nahezu alle Branchen der industriellen Fertigung, der gewerblichen Wirtschaft, in den Dienstleistungssektor, (im Aktienhandel, im Versandhandel usw.), und folglich auch in die Berufs- und Arbeitswelt (Ramge 2018). Der Einsatz Künstlicher Intelligenz ist schon längst nicht mehr wegzudenken aus dem Medienbetrieb, dem Personen- und Güterverkehr (Auto-, Schiffs- und Luftverkehr), aus der Logistik, dem Wohnungswesen und dem Gesundheitswesen ${ }^{11}$ (namentlich in der klinischen Diagnostik, Prothetik und Radiologie), aus der Wettervorhersage, dem Klima- und Umweltschutz. Aber auch bei der Bearbeitung von Standardfällen in Anwaltskanzleien, auf dem Gebiet der Inneren Sicherheit, insbesondere bei polizeilichen Maßnahmen der Gefahrenvorsorge und der Gefahrenabwehr, ferner bei der Produkterkennung, bei der Spracherkennung, der Bilderkennung, in Übersetzungsbüros usw. ist der Einsatz Künstlicher Intelligenz schon längst unverzichtbar geworden. Mit einem Wort: es gibt kaum noch einen Lebensbereich in Gesellschaft, Wirtschaft und Staat, wo Künstliche Intelligenz nicht zur Anwendung kommt, und das mit exponentiell steigendem Tempo. Dabei tendiert die Entwicklung zur Vernetzung, Kommunikation und Interaktion der

\footnotetext{
${ }^{10} \mathrm{Als}$ Einstieg siehe den Wikipedia Artikel: Industrie 4.0: https://de.wikipedia.org/wiki/ Industrie_4.0.; zur aktuellen Lage aus deutscher Sicht siehe das Interview mit Martin Rutkowski (2019), sowie Schmidhuber (2018) und ebenso der PC-Linguist Assadollahi (2018).

${ }^{11}$ Über weitere Perspektiven des Einsatzes von KI im Gesundheitswesen siehe aus jüngster Zeit (Nosthoff und Maschewski 2019; Spehr 2019; Langkafel 2015) Sehr umstritten ist der Einsatz von KI in der Pflege. Zu diesem namentlich in Japan forcierten Kurs siehe Maak (2018).
} 
Anwendungsbereiche. Bekannte Beispiele sind solche integrierten Systeme wie das Smart Home im Kleinen, die Smart City (Graz) im Großen sowie, ganz allgemein, das heute erst im Entstehen begriffene „Internet der Dinge“ (vgl. Lenzen 2018a, b, S. 181 ff.).

Die beschleunigte Ausweitung der Anwendung von Künstlicher Intelligenz hat in Deutschland und in der EU die Forderung aufkommen und lauter werden lassen, die ethischen und rechtlichen Bindungen bei der Entwicklung und Anwendung „künstlicher Intelligenz“ verstärkt zu berücksichtigen und so früh und so weit wie möglich zu integrieren. Die maßgebenden nationalen und internationalen Leitlinien der auf dem Gebiet der Robotik und Künstlicher Intelligenz formulierten Ethik-Grundsätze sind dabei nicht bereichsspezifisch nach Branchen oder gesellschaftlichen Lebensbereichen aufgegliedert, sondern einheitliche Vorgaben und Anforderungen, die sich auf den Einsatz von Künstlicher Intelligenz im Allgemeinen beziehen.

\section{Dimensionen der Verantwortung und Versuche ihrer ethischen Operationalisierung in der EU}

Man kann die Ethik-Grundsätze auf den Generalnenner bringen, dass sie die Verantwortung des Menschen im Umgang mit Künstlicher Intelligenz und Robotik konkretisieren und operationalisieren.

Verantwortung hat, wie der Sprachgebrauch zeigt, sowohl in individueller als auch in kollektiver Hinsicht eine Doppelpoligkeit (vgl. Picht 1967, S. 327 ff.): Verantwortung besteht erstens vor einem Subjekt oder einer Instanz sowie zweitens für etwas oder auch für jemand, d. h. entweder für ein Objekt, eine Sache oder eine Aufgabe oder aber für ein Subjekt. Welches die Subjekte sind, vor denen die Verantwortung besteht, muss für jede Lage besonders bestimmt werden. Fragt man, vor wem ein Individuum, z. B. der auf dem Gebiet der Künstlichen Intelligenz tätige Ingenieur, für sein Tun verantwortlich ist, dann darf im säkularen Staat als Antwort nicht auf eine religiöse Instanz, also auf Gott, verwiesen werden, sondern nur auf eine weltliche Instanz. Im demokratischen Rechtsstaat der Bundesrepublik Deutschland fällt der Blick auf das Grundgesetz, also die Verfassung, und hier auf die Grundrechte, beginnend mit der Menschenwürde (Art. 1 Abs. 1 GG). Die Antwort auf die Frage, vor wem die Verantwortung besteht, würde demnach lauten: vor den Trägern der Menschenwürde, also vor den Mitmenschen, und stellvertretend für diese vor der staatlichen Gemeinschaft, also vor dem Staat repräsentiert durch die Verfassungsorgane. 
Als Antwort auf den Staat zu verweisen, in dem die betreffende Person als Trägerin der Verantwortung lebt und arbeitet, hat den Vorzug, dass die Person den Sanktionen unterliegt, welche die Gesetze der entsprechenden Verfassungs- und Rechtsordnung vorsehen, wenn die Verantwortung nicht wahrgenommen wird.

Die Antwort auf die Frage, für wen oder was eine Person Verantwortung trägt, wenn es um das Zusammenwirken lebender und nichtlebender Entitäten unter den Bedingungen des digitalen Zeitalters geht, lässt sich ebenfalls aus den Grundrechten des Grundgesetzes, konkret aus der Garantie der Menschenwürde ableiten. Die Antwort lautet dann: der auf dem Gebiet der Künstlichen Intelligenz tätige Ingenieur ist dafür verantwortlich, dass er im Zusammenwirken mit Künstlicher Intelligenz die Menschenwürde nicht antasten darf. Anders formuliert, er muss Vorsorge dafür treffen, dass das von Künstlicher Intelligenz gesteuerte Zusammenwirken lebender und nichtlebender Entitäten nicht in die Würde des Menschen eingreift.

Außer der Menschenwürde kommen noch andere Grundrechte in Betracht, für deren Unantastbarkeit die betreffende Person Verantwortung trägt, nämlich dass Diskriminierungen aus den in Art. 3 Abs. 3 GG $^{12}$ genannten Gründen ausgeschlossen sind, dass nicht in die Privatsphäre eingegriffen wird, dass die persönliche Freiheit von niemand eingeschränkt wird usw.

Die Bundesregierung hat im Juli 2018 Eckpunkte für eine Strategie Künstliche Intelligenz (Bundesregierung 2018) veröffentlicht und darin außer einer Darstellung der Ausgangslage Kataloge von Zielen und Anwendungsbereichen (Handlungsfelder) von Künstlicher Intelligenz aufgestellt, unter denen Wirtschaft, Arbeit und Bildung an vorderer Stelle stehen ${ }^{13}$. Das Papier ist im Zusammenhang mit der 2016 von der EU erlassenen, seit Mai 2018 anzuwendenden und unmittelbar in allen EU-Mitgliedsstaaten geltenden Datenschutz-Grundverordnung $\mathrm{zu}$ sehen, die dem Schutz der personenbezogenen Daten dient und die bislang geltenden nationalen Vorschriften über die Erhebung, Speicherung, Verarbeitung und Löschung von Daten überwölbt, vereinheitlicht, modernisiert und dabei teilweise

\footnotetext{
${ }^{12}$ Namentlich in den USA hat sich schon vor Jahren herausgestellt, dass der Einsatz von KI und Algorithmen u. a. im Personalwesen von Unternehmen wegen der unkritischen Reproduktion von gruppenbezogenen Stereotypen und Vorurteilen zu verfassungswidrigen Diskriminierungen führen kann. Lobe (2019); ferner ein Interview mit Francesca Rossi von IBM (Budras, 2018) und Beck (2019).

${ }^{13}$ Ein Kommentar dazu von Armbruster (2018a).
} 
geändert hat ${ }^{14}$. Der Schutz erstreckt sich nicht nur auf alle Informationen identifizierter (natürlicher) Personen, sondern auch auf Informationen (Daten) identifizierbarer Personen. Identifizierbar ist eine natürliche Person (Art. 4), „die direkt oder indirekt, insbesondere mittels Zuordnung zu einer Kennung wie einem Namen, zu einer Kenn-Nummer, zu Standortdaten, zu einer Online-Kennung oder [die Zuordnung] zu einem oder mehreren besonderen Merkmalen [identifiziert werden kann], welche Ausdruck der physischen, physiologischen, genetischen, psychischen, wirtschaftlichen, kulturellen oder sozialen Identität dieser natürlichen Person sind“" (Europäisches Parlament 2016, Art. 4).

Es ist offenkundig, dass dieser detaillierte Katalog von personenbezogenen Merkmalen, deren Zusammenführung die namentliche Bestimmung von Personen ermöglicht, in einem starken Spannungsverhältnis zum Einsatz Künstlicher Intelligenz steht. Umso wichtiger ist die Frage, ob sich die Nutzer von Künstlicher Intelligenz auf eine Erlaubnis zur Verarbeitung personenbezogener Daten berufen können. Die Verarbeitung personenbezogener Daten ist grundsätzlich nur mit Erlaubnis zulässig (Art. 6). Sie erfolgt naturgemäß an erster Stelle durch die Einwilligung der Person, um deren Daten es geht. Sie ist darüber hinaus aber auch aus einer Reihe von sonstigen legitimen Gründen zulässig, z. B. wenn die Datenverarbeitung erforderlich ist, um lebenswichtige Interessen zu schützen, oder wenn die Datenverarbeitung der Erfüllung einer Aufgabe dient, die im öffentlichen Interesse liegt.

Ob die Datenschutz-Grundverordnung kraftvoll genug ist, um die Möglichkeit der mittelbaren Identifizierung einer natürlichen Person durch Zusammenführung von im Prinzip unproblematischen Daten auszuschließen, kann zweifelhaft sein ${ }^{15}$. Gänzlich unklar ist, ob bzw. wie eine betroffene Person überhaupt etwas über ihre durch Einsatz von Künstlicher Intelligenz bewirkte Identifizierung erfährt und erfahren kann. Es ist denn auch kritisch vermerkt worden, dass sich die Datenschutz-Grundverordnung mit den spezifischen Problemen und Gefahren gar nicht befasse, die mit dem Einsatz Künstlicher Intelligenz verbunden seien, nämlich mit den sozialen Netzwerken, mit der Datenflut von Big Data und ihrer Auswertung, mit Suchmaschinen, Cloud-Computing, Ubiquitous Computing usw.

\footnotetext{
${ }^{14}$ Europäisches Parlament (2016) Quelle: https://www.computerundrecht.de/ OJ_L_2016_119_FULL_DE_TXT.pdf (Abgerufen am 10.10.2019). Zum Inhalt und zu ersten Kommentierungen der Datenschutz-Grundverordnung siehe zu einer ersten Orientierung den Wikipedia-Eintrag zur Datenschutz Grundverordnung. Quelle: https://de.wikipedia.org/wiki/ Datenschutz-Grundverordnung (Abgerufen am 10.10.2019).

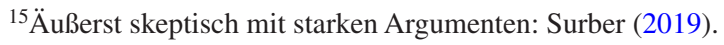


In zwei Punkten stellt sich die Datenschutz-Grundverordnung immerhin der Verantwortung für eine Abwehr von Missbrauch, und zwar durch die beiden Ansätze von Datenschutz durch Technikgestaltung: erstens durch Privacy by Design (Wikipedia - Privacy by Design o. J.b, Abgerufen: 10.10.2019), d. h. durch die Berücksichtigung ethischer Grundsätze in Künstliche-Intelligenz-Verarbeitungsprozessen von Anfang an, und zweitens durch Privacy by Default (Datenschutzbeauftragter INFO 2017), d.h. durch Erhebung des Datenschutzes (Vertraulichkeit) zum technischen Standard im Interesse der Nutzer (Digitalcourage e. V. 2014).

Die dem deutschen Grundgesetz entnommenen normativen Maßstäbe für die Erfüllung der Anforderungen, die sich aus der Verantwortung der an der Entwicklung von Künstlicher Intelligenz arbeitenden Spezialisten ergeben, liegen in der Sache auch den Ethik-Leitlinien für eine vertrauenswürdige Künstliche Intelligenz zugrunde, deren Entwurf eine sogenannte Hochrangige Expertengruppe für Künstliche Intelligenz im Auftrage der EU-Kommission am 18. Dezember 2018 veröffentlicht hat (European Commission 2018) ${ }^{16}$. Die Leitlinien sind, wie vorgesehen, im April 2019 von der Expertengruppe überarbeitet worden (Hillmer 2019) und sollen nach dem Plan der EU-Kommission im Sommer 2019 einer breiten öffentlichen Diskussion unterzogen werden. Künstliche Intelligenz Made in Europe orientiert sich an der EU-Grundrechte-Charta vom 7. Dezember 2000. Die Charta verkündet Menschenwürde, Freiheit, Gleichheit und Solidarität und räumt den Grundrechten der Privatsphäre und des Diskriminierungsverbots, die bei der Anwendung von Künstlicher Intelligenz, wie die Erfahrung zeigt, besonders gefährdet sind, hohen Rang ein. Kein Zweifel: Der Schutz der europäischen Grundwerte hat für die Bewahrung der Würde des Menschen als Menschen überragende Bedeutung, denn letztlich geht es bei der Verantwortung für jene Werte um nicht weniger als um den Schutz der Menschheit vor der Gefahr ihrer Herabwürdigung zum Objekt unter das Diktat eines Roboters nach Maßgabe Künstlicher Intelligenz (vgl. Picht 1967) ${ }^{17}$. Das zwingt gleichsam die EU, stellvertretend für Europa insgesamt, einen eigenen, an jenen höchsten Grundwerten orientierten ethischen Kodex für Künstliche Intelligenz zu erstellen. Er bündelt sich in dem Kriterium der Vertrauenswürdigkeit. Es soll nach dem Wunsch der EU-Kommission - die Abgrenzung vor allem zur Volksrepublik

\footnotetext{
${ }^{16}$ In Deutsch existiert nur eine offizielle (5-seitige) ,Zusammenfassung“.

${ }^{17}$ Wir sehen hier den Fall einer Konstellation, die Georg Picht vor Jahrzehnten (1967) mit Blick auf die Möglichkeit eines Nuklearkrieges als den Fall ,einer universalen Verantwortung der Menschheit“ beschrieben hat, die aber auch jeden einzelnen Menschen in „verantwortlicher Stellung“" trifft.
} 
China ist unüberhörbar - das Markenzeichen der Künstlichen Intelligenz Made in Europe sein.

Nach der Vorstellung des Entwurfes der Ethik-Leitlinien gilt Künstliche Intelligenz dann als vertrauenswürdig, wenn sie erstens auf den Menschen ausgerichtet ist (menschenzentriert) und zweitens die folgenden ethischen Grundsätze befolgt und fördert: Gutes tun, keinen Schaden zufügen, menschliche Autonomie und Gerechtigkeit achten. Zusätzlich betonen die Leitlinien, die Vertrauenswürdigkeit der Künstlichen Intelligenz hänge entscheidend davon ab, dass aufgrund der Transparenz der von Künstlicher Intelligenz gesteuerten Prozesse der Bürger über mögliche Folgen und Risiken der Künstlichen Intelligenz informiert sei. Transparenz verlange, dass die mithilfe Künstlicher Intelligenz gefundenen Ergebnisse und getroffenen Entscheidungen erklärbar, nachvollziehbar, rückverfolgbar und nach Maßgabe der ethischen Werte auch überprüfbar seien.

Das Konzept mag, zumindest auf den ersten Blick, überzeugend wirken, doch haftet ihm eine bei näherem Hinsehen sichtbar werdende zweifache Schwäche an: sie betrifft sowohl die vier materiellen, dem Axiom der Menschenzentriertheit dienenden ethischen Grundsätze, als auch die vier formellen Verfahrensgrundsätze, welche die zum prozessualen Leitprinzip erhobene Transparenz der Algorithmen gewährleisten sollen, die die zu fällenden Entscheidungen vorbereiten oder bewirken.

Das Axiom der Menschenzentriertheit und die vier das Axiom implementierenden ethischen Grundsätze suggerieren eine Eindeutigkeit und Identität, die in ethischer Hinsicht nicht besteht und auch nicht bestehen kann; denn was ist „das Gute“, was man tun soll und was ist ein Schaden, den man vermeiden soll, und was bedeuten Autonomie und menschliche Gerechtigkeit? Alles das sind Begriffe, deren Inhalte sich seit der Antike immer wieder gewandelt haben und die nach wie vor heftig umstritten sind. Infolgedessen mangelt es ihnen an jener Klarheit, die nach dem Wunsch der EU die Ethik-Leitlinien Made in Europe auszeichnen sollen.

Dazu tritt ein weiterer Einwand: die vier ethischen Grundsätze sind derartig abstrakt, aber auch banal formuliert und bewegen sich in einer solchen luftigen Höhe, dass ihre Eignung und Operationalisierbarkeit als kritischer Maßstab für die Einschätzung und eventuelle Verwerfung von Algorithmen und Produkten Künstlicher Intelligenz höchst zweifelhaft erscheinen muss.

Erhebliche Skepsis dürfte nicht weniger hinsichtlich der Realisierbarkeit von Transparenz angebracht sein. Gewiss ist es richtig, dass Transparenz als verfahrensmäßiges Leitprinzip wegen ihrer organischen Verknüpfung mit dem Prinzip der Öffentlichkeit erfahrungsgemäß eine entscheidende Voraussetzung 
dafür ist, dass die Einhaltung der vier materiellen Ethik-Grundsätze durch interessierte Bürger wenigstens im Ansatz kontrolliert werden kann; und es hört sich auch wohlklingend an, dass die mithilfe Künstlicher Intelligenz gefundenen Ergebnisse und getroffenen Entscheidungen erklärbar, nachvollziehbar, rückverfolgbar und nach Maßgabe der ethischen Werte tatsächlich auch überprüfbar sein sollen $^{18}$. Aber die vier Verfahrensgrundsätze sind äußerst anspruchsvolle Vorgaben und Erwartungen, die nicht im Einklang mit der in der Literatur immer wieder zu lesenden Klage stehen, dass die bei künstlicher Intelligenz zum Einsatz kommenden Algorithmen erstens nicht selten Geschäftsgeheimnis seien und dass zweitens die Operationen der Künstlichen Intelligenz so schnell und komplex abliefen, dass auch Experten von einer undurchdringlichen Black Box sprächen (Betschon 2018, 2019b; Budras 2019; Manuela Lenzen 2018a, b).

Zwar gibt es Stimmen, die jene Schwierigkeiten für überwindbar und die Probleme für lösbar halten (Gigerenzer et al. 2018), aber die von ihnen vorgeschlagenen Verfahren sind äußerst kompliziert und setzen aufseiten der Zivilgesellschaft und der Bürger Entschlossenheit und Hartnäckigkeit voraus, die versprochene Transparenz im Alltag auch durchzusetzen. Eine solche Annahme erscheint jedoch allzu idealistisch und deswegen wenig realistisch.

Die Hochrangige Expertengruppe hat, wie es scheint, die Kritik an ihrem Entwurf von Ethik-Richtlinien aufgegriffen und das Hauptziel einer europäischen Strategie, nämlich die Vertrauenswürdigkeit von Künstlicher Intelligenz, durch die folgenden sieben Leitziele operationalisiert, die offenkundig die materiellethischen und die formell-verfahrensmäßigen Grundsätze des ersten Entwurfs integrieren (European Commission 2019, Abgerufen am 10.10.2019 von https:// ec.europa.eu/futurium/en/ai-alliance-consultation/guidelines) ${ }^{19}$ :

1. „Vorrang menschlichen Handelns und menschlicher Aufsicht: KI-Systeme sollten gerechten Gesellschaften dienen, indem sie das menschliche Handeln und die Wahrung der Grundrechte unterstützen; keinesfalls aber sollten sie die Autonomie der Menschen verringern, beschränken oder fehlleiten.

2. Robustheit und Sicherheit: Eine vertrauenswürdige KI setzt Algorithmen voraus, die sicher, verlässlich und robust genug sind, um Fehler oder

\footnotetext{
${ }^{18}$ Kritisch dazu Yogeshwar (2019) und Gillen (2019a, b); dagegen (Ala-Pietilä 2019). Der Autor ist Vorsitzender der EU-Beratungskommission, die den Entwurf der „Leitlinien“ ausgearbeitet hat.

${ }^{19}$ Diese Grundsätze wurden am 8. April 2019 veröffentlicht (European Commission 2019). Einen ersten Kommentar liefert Gillen (2019b).
} 
Unstimmigkeiten in allen Phasen des Lebenszyklus des KI-Systems zu bewältigen.

3. Privatsphäre und Datenqualitätsmanagement: Die Bürgerinnen und Bürger sollten die volle Kontrolle über ihre eigenen Daten behalten, und die sie betreffenden Daten sollten nicht dazu verwendet werden, sie zu schädigen oder zu diskriminieren.

4. Transparenz: Die Rückverfolgbarkeit der KI-Systeme muss sichergestellt werden.

5. Vielfalt, Nichtdiskriminierung und Fairness: KI-Systeme sollten dem gesamten Spektrum menschlicher Fähigkeiten, Fertigkeiten und Anforderungen Rechnung tragen und die Barrierefreiheit gewährleisten.

6. Gesellschaftliches und ökologisches Wohlergehen: KI-Systeme sollten eingesetzt werden, um einen positiven sozialen Wandel sowie die Nachhaltigkeit und ökologische Verantwortlichkeit zu fördern.

7. Rechenschaftspflicht: Es sollten Mechanismen geschaffen werden, die die Verantwortlichkeit und Rechenschaftspflicht für KI-Systeme und deren Ergebnisse gewährleisten.“

Die EU-Kommission versteht die Ethik-Leitlinien als ein living document und beabsichtigt dementsprechend, über sie im Jahre 2020 weiter zu beraten und dann über das abschließende Vorgehen zu entscheiden.

Aus der Perspektive der Verantwortung für den ethischen Umgang mit Künstlicher Intelligenz hat Christoph Markschies ${ }^{20}$ einen prinzipiellen Einwand gegenüber dem Entwurf der „EU-Ethik-Richtlinien“ erhoben, der die Stimmigkeit des Konzepts infrage stellt und auch in Bezug auf die Neufassung der Richtlinien seine Bedeutung nicht eingebüßt hat (Markschies 2019). Markschies' Kritik setzt bei dem von der EU als zentral herausgestellten Ziel der vertrauenswürdigen Künstlichen Intelligenz (truthworthy artficial intelligence) an. Unter Einbeziehung (und Ablehnung) des vom Massachusetts Institute of Technology (MIT) vertretenen Konzepts einer Moral Machine hebt Markschies (2019) hervor, dass vertrauenswürdig, streng genommen, nicht eine Technologie sein könne, sondern nur die hinter ihr stehenden Menschen. Vertrauenswürdig (oder nicht) seien daher ,nicht Systeme der Künstlichen Intelligenz, sondern die Menschen,

\footnotetext{
${ }^{20}$ Der Theologe Christoph Markschies, 2006 bis 2011 Präsident der Humboldt-Universität Berlin, ist Sprecher der interdisziplinären Arbeitsgruppe „Verantwortung: Maschinelles Lernen und Künstliche Intelligenz" der Berlin-Brandenburgischen Akademie der Wissenschaften.
} 
die sie programmieren, und die, die für rechtlich geordnete Zulassungsverfahren Verantwortung tragen" (Markschies 2019, FAZ, S. 11). Hinsichtlich der Künstlichen Intelligenz hat der Einwand ein besonders hohes Gewicht, weil in ihrem Falle die für verbindlich erklärten rechtlichen Grundwerte, ethischen Grundsätze und Standards über Algorithmen in Entscheidungsprozesse eingespeist werden, Algorithmen, die als solche schon deswegen keine Vertrauenswürdigkeit beanspruchen können, weil das nicht nur die völlige Transparenz ihrer Entstehung, also der digitalen Programmierung, sondern auch eine ethisch-rechtliche Fachkontrolle der für den Einsatz bestimmten Algorithmen voraussetzen würde. An erster Stelle aber müsste das, so Markschies (2019), ethische Bildung derjenigen voraussetzen, welche Programme für Künstliche Intelligenz schreiben, und für diejenigen, welche über deren praktischen Einsatz entscheiden. Auch seien rechtlich normierte Verfahren notwendig, wenn man Programme daraufhin überprüfen wolle, ob sie bestimmten ethischen Normen und rechtlichen Standards entsprechen (Markschies 2019).

Alle diese Voraussetzungen existieren bestenfalls sektoral und allenfalls in Ansätzen.

Man muss daraus den Schluss ziehen, dass das von der EU verkündete Ziel, in ihrem Herrschaftsbereich für vertrauenswürdige Künstliche Intelligenz zu sorgen, noch in weiter Ferne liegt, wenn es denn überhaupt erreichbar und mehr als eine schöne Vision und ethisch-politische Utopie sein sollte. Die Dimensionen der Zielsetzung, die Komplexität des Phänomens der Künstlichen Intelligenz und die hohe Dynamik ihrer Entwicklung und Veränderung geben wenig Anlass zu der Hoffnung, dass sich die ethischen und juristischen Anforderungen an die Verantwortung gegenüber der Künstlichen Intelligenz durchsetzen werden.

\section{$4 \quad$ Beispiele der Reichweite von juristischer Verantwortung}

Die Reichweite von Verantwortung kann sich zwar im Prinzip auf beide Dimensionen, kann sich also sowohl auf die Verantwortung vor jemandem als auch auf die Verantwortung für jemanden oder für etwas erstrecken, aber das wirklich brisante Problem ist hier die Frage, wie weit reicht die Verantwortung für, abstrakt gesprochen, Personen oder/und Sachen? Es liegt auf der Hand, um eine allgemeine, grundsätzliche Feststellung vorweg zu treffen, dass Verantwortung nicht unbegrenzt sein kann. Wie Georg Picht (1967, S. 334 f.) zutreffend herausgearbeitet hat, kann Verantwortung einer Person nur so weit reichen, wie sie zuständig ist und Kraft ihrer Zuständigkeit über die Macht und 
die Werkzeuge verfügt, um auf das Geschehen in ihrem Verantwortungsbereich effektiv einwirken zu können.

Die Zuständigkeit und Ermächtigung, Entscheidungen und Maßnahmen zu treffen, werden bei individueller und bei kollektiver Verantwortung unterschiedlich ausgeprägt sein und unterschiedliche Reichweite haben. Denn indem Träger von kollektiver Verantwortung auch eine Gebietskörperschaft wie eine Gemeinde, ein Bundesland oder die Bundesrepublik Deutschland insgesamt sein kann, ist die Reichweite ihrer Verantwortung entsprechend der Kompetenzordnung im Bundesstaat sehr verschieden.

Bei der uns hier interessierenden Frage nach der Reichweite der Verantwortung (im Falle der Entwicklung und Anwendung von Künstlicher Intelligenz) für die Integrität der humanen Grundwerte, Schutzgüter und Grundrechte, namentlich von Menschenwürde und Autonomie, richten sich die Augen zwangsläufig auf Wirtschaftsunternehmen sowie auf Forschung und Wissenschaft, aber auch auf den Staat, der als Gesetzgeber, als Verwaltung und Justiz die Beachtung und Durchsetzung jener rechtlichen und ethischen Standards letztlich verbürgt und zu garantieren hat.

Es liegt auf der Hand, dass wegen der außerordentlichen Komplexität des mit der Entwicklung und Anwendung von Künstlicher Intelligenz betroffenen Lebensverhältnisse von Gesellschaft, Wirtschaft und Staat die Reichweite der kollektiven Verantwortung ganz dominant im Vordergrund steht und daher unsere besondere Aufmerksamkeit erheischt. Ebenso dürfte es unmittelbar einleuchten, dass die Reichweite der Verantwortung nicht abstrakt und für alle Akteure gleich bestimmt und fixiert werden kann, sondern sich sehr verschieden darstellt, abhängig zum Beispiel von der Weite des geplanten Anwendungsbereichs der Künstlichen Intelligenz.

Nehmen wir als Beispiel das vieldiskutierte autonome Fahren, nicht unbedingt die Umstellung des gesamten Straßen- bzw. Autoverkehrs auf Autopiloten, sondern nur den Verkehr auf Autobahnen (Hilgendorf et al. 2015; Lutz 2015; Feldle 2018; Scherff 2018). Trotz der darin liegenden Begrenzung würden wir es mit einem höchst komplexen, sich auf das ganze Staatsgebiet erstreckenden Verkehrssystem zu tun haben, das Auswirkungen auf sämtliche Lebensbereiche der Gesellschaft und der Einzelnen besäße. Die Entscheidung über seine Einführung läge daher beim Staat in Gestalt des Bundesgesetzgebers.

Es ist evident, dass sich davon die Umrüstung einer Villa mit Hilfe von Künstlicher Intelligenz in ein sogenanntes smart home wesentlich unterscheidet.

Bevor der Gesetzgeber seine Verantwortung wahrnehmen könnte, über die Einführung des autonomen Fahrens zu entscheiden, wären die Fahrzeuge mit solchen Vorrichtungen Künstlicher Intelligenz auszurüsten, die erstens 
ein autonomes Fahren ermöglichen und zweitens die Probleme eines sicheren Straßenverkehrs lösen würden. Die Verantwortung dafür läge bei den darauf spezialisierten Firmen und ihren Mitarbeitern, aber auch bei den staatlichen Behörden, die über die Zulassung der Fahrzeuge zu entscheiden hätten. Es ergibt sich das Bild einer gestuften Verantwortungskette, an deren Spitze der Gesetzgeber zu denken wäre, der die endgültige Entscheidung über die Freigabe und Einführung des Systems zu treffen hätte.

Bekanntlich ist immer wieder die Frage diskutiert worden, wer zur Verantwortung zu ziehen ist, wenn im autonomen Betrieb des Verkehrs sich Unfälle ereignen, dabei auch Menschen zu Schaden kommen und unter Umständen getötet werden. Vorauszuschicken ist, bevor wir uns rechtlichen Details zuwenden, die grundsätzliche Feststellung, dass der mit künstlicher Intelligenz ausgestattete Roboter im Unterschied zum Menschen nicht schuldfähig ist. Ihm kann deswegen ein von seinem technischen Versagen verursachtes schädigendes Verhalten nicht zugerechnet und daher auch nicht vorgeworfen werden. Der Roboter ist nicht verantwortlich; verantwortlich kann nur der Mensch sein, d. h. eine natürliche Person sowie unter Umständen auch eine juristische Person, weil hinter ihr Menschen stehen und sie durch Menschen handelt.

Das bedeutet jedoch keineswegs und kann auch nicht bedeuten, dass für den von einem Roboter verursachten Schaden niemand verantwortlich gemacht werden kann. Die Realisierung der Verantwortung ist im Rahmen unserer Rechtsordnung sehr wohl möglich (Hilgendorf et al. 2015; Lutz 2015; Feldle 2018; Scherff 2018). Es bieten sich hier verschiedene Lösungen an. Teils wird auf der Grundlage des geltenden Rechts vorgeschlagen, den Halter (Eigentümer) des den Unfall verursachenden ,autonom“ bewegenden Fahrzeugs für den Schaden haften zu lassen. Eines der Probleme, die sich hier stellen, ist der Umstand, dass den Halter keine Schuld an dem Unfall trifft. Man käme aber leicht darüber hinweg, indem man den Halter nach den Grundsätzen der sogenannten Gefährdungshaftung zur Verantwortung ziehen würde. Das bedeutet, dass seine Haftung für den Unfall anknüpfen würde an die schlichte Tatsache, dass er sich in den Straßenverkehr mit seinem Auto begeben und dadurch eine für den Unfall entscheidende Ursache gesetzt hat.

Eine andere Möglichkeit wäre, die autonom fahrenden Fahrzeuge rechtlich als Roboter im Wege der Fiktion per Gesetz zu juristischen Personen eigener Art (sui generis) zu machen ${ }^{21}$. Die Haftung könnte man dann auf zweierlei Weise recht-

\footnotetext{
${ }^{21}$ Eingehend $\mathrm{zu}$ den möglichen juristischen Konstruktionen von Alternativen (Günther 2016, S. 45 ff.).
} 
lich konstruieren: erstens könnte man den Eigentümer für den eRoboter nach den zivilrechtlichen Regeln über den Erfüllungs- oder Verrichtungsgehilfen oder über den Besitzdiener (d. h. als selbständig Werkzeug) haften lassen. Der Gesetzgeber könnte aber, zweitens, auch bestimmen, dass der eRoboter als juristische Person selbstständig haftet. Das würde und müsste die Konsequenz haben, den Roboter der Haftpflichtversicherung zu unterwerfen und ihn auf der Grundlage haften zu lassen. Das wäre die zivilrechtliche Haftung, die sich in vollem Umfange auf den Schaden erstrecken würde.

Kein unüberwindliches Problem würde die polizeirechtliche Haftung aufwerfen, denn in diesen Fällen könnte man den Eigentümer des eRoboters über die polizeiliche Zustandsverantwortung, d.h. seine Haftung für den ordnungsgemäßen Zustand seiner Sachen zur Verantwortung ziehen.

Schwieriger wäre die Lösung des Problems einer eventuellen strafrechtlichen Verantwortung, z. B. wegen Tötung im Zuge eines Verkehrsunfalls. Den Vorwurf der Fahrlässigkeit oder gar des Vorsatzes könnte man dem Fahrzeug nicht machen, weil das die Schuldfähigkeit voraussetzen würde, die der Roboter aber nicht hat. Man könnte daran denken, den Halter und Eigentümer des Fahrzeuges strafrechtlich zu belangen und ihn wegen fahrlässiger Tötung zur Verantwortung zu ziehen. Das würde nach geltendem Strafrecht erstens voraussetzen, dass er mit seinem Verhalten eine Kausalkette begründet hat, die zu dem Unfall geführt hat, und dass er zweitens eine Sorgfaltspflicht verletzt hat und es deswegen zu dem Unfall mit Todesfolge gekommen ist. Den Vorwurf der Sorgfaltspflichtverletzung wird man dem Halter und Eigentümer des autonom fahrenden Autos aber gerade nicht machen können, denn sein Eingreifen in das Verkehrsgeschehen ist gerade nicht vorgesehen und im Prinzip unzulässig. Der Jurist wäre hier mit seinem Latein gleichwohl nicht am Ende. Denn entweder könnte man durch Gesetz eine strafrechtliche Haftung definitiv ausschließen oder man könnte per Gesetz gewissermaßen eine strafrechtliche Gefährdungshaftung einführen, deren Sanktion zwar nicht auf Freiheitsentzug, wohl aber auf eine Geldstrafe zulasten des Eigentümers und Halters lauten könnte. Der Unterschied zur zivilrechtlichen Gefährdungshaftung bestünde allein in dem moralischen Vorwurf an die Person des Eigentümers und Halters, um dessen Letztverantwortung aufzuzeigen und den Angehörigen des Opfers eine gewisse Genugtuung zu verschaffen.

Unter Juristen, die mit Robotik befasst sind, wird diskutiert, ob es sinnvoll und vielleicht sogar geboten sein könnte, den Roboter zu einer juristischen Person zu machen. Die ePerson des Roboters würde dann zu einem Haftungssubjekt, für das - kraft Gesetzes - eine Versicherung abzuschließen wäre, um seine Haftung nicht leer laufen zu lassen. Diese sich an der juristischen Person orientierende Konstruktion erscheint aber nur dann sinnvoll und erforderlich, wenn der Roboter 
unter dem Gesichtspunkt kausaler Geschehensabläufe so weit von einer eindeutig als verantwortlich zu identifizierenden, sei es natürlichen oder juristischen Person (Unternehmen usw.) entfernt ist, dass eine andere Möglichkeit der Haftung praktisch ausgeschlossen ist. Das geht nur über eine Gefährdungshaftung, weil der Roboter schuldunfähig ist, oder durch eine Schuldfiktion zulasten des Roboters, der dann allerdings wie ein Mensch und wie eine der Verantwortung fähige Person behandelt würde. Das wäre ein Präzedenzfall der juristischen Vermenschlichung des Trägers künstlicher Intelligenz.

\section{Schlussbemerkung}

Die Entwicklung Künstlicher Intelligenz ist ein globaler Prozess, an dessen Anfang die Menschheit erst steht. Sie stellt eine technische Entwicklungsstufe in der Geschichte des Menschen dar, die mit der Einführung der Elektrizität verglichen worden ist (vgl. Assadollahi 2018) ${ }^{22}$. Das ist insofern richtig, als die Elektrizität als Energiequelle den Alltag des Menschen in allen seinen Dimensionen weltweit mitbestimmt. An dem Vergleich zeigt sich aber ein wesentlicher und für die weitere Geschichte des Menschen und der Menschheit ganz entscheidender Unterschied: die Künstliche Intelligenz ist kein statisches Phänomen, sondern ein Prozess von hoher Dynamik, in dessen Verlauf Künstliche Intelligenz in Gestalt von Robotern die Fähigkeit erwirbt, den Menschen aus gewissen Bereichen zu verdrängen und zu ersetzen.

Für eine Hauptgefahr der Entwicklung halte ich, dass der Mensch, fasziniert von der Leistungsfähigkeit der Künstlichen Intelligenz, ein am Roboter orientiertes und damit reduktionistisches Menschenbild übernimmt und das Humanum des Menschen verrät ${ }^{23}$. Die Gefahr, die von Künstlicher Intelligenz für den Menschen ausgeht, ist nach meiner Überzeugung nicht die, dass Roboter den Menschen überflügeln und den Menschen in der vom Menschen gemachten Welt gleichsam hinter sich lassen werden, sondern dass der Mensch sich den Roboter, sein Werkzeug, zum Vorbild nimmt, sich selbst funktionalisiert und in einseitiger, auf Effektivität ausgerichtete Weise „optimiert“ (vgl. Schwartmann

\footnotetext{
${ }^{22}$ So z. B. der Google-Vorsitzende, Sundai Pichai, im Interview in der FAZ (Assadollahi 2018).

${ }^{23}$ Von einer solchen Entwicklung scheint, zumindest im Ergebnis, der Historiker Yuval Harari überzeugt zu sein. Siehe Lobe (2018).
} 
2018) ${ }^{24}$. Diese Gefahren lassen sich nur bezwingen, wenn die Entwicklung und Anwendung künstlicher Intelligenz auf das beschränkt bleiben, was die Künstliche Intelligenz ist, nämlich ein die Menschheit nicht bedrohendes, sondern den Menschen helfendes Werkzeug und Instrument. Die Überzeugung von der Wahrheit und Richtigkeit dieses ethischen Standpunktes und dieser moralischen Grundhaltung muss aber von der Bereitschaft und dem Willen einer kritischen Menge von Menschen getragen sein, sich aktiv für die Erhaltung der Menschheit gegenüber allen Bedrohungen und Gefährdungen einzusetzen (vgl. Strasser 2019).

\section{Literatur}

Ala-Pietilä, P. (30. Januar 2019). Vertrauenswürdige Künstliche Intelligenz. Frankfurter Allgemeine Zeitung (FAZ), 11.

Ankenbrand, H. (27. März 2019). Deutschland kleckert - China klotzt. Frankfurter Allgemeine Zeitung (FAZ), 17.

Armbruster, A. (25. Juli 2018a). Künstliche Intelligenz made in Germany. Frankfurter Allgemeine Zeitung (FAZ), 15.

Armbruster, A. (6. November 2018b). Interview mit Yann LeCun, KI-Spezialist bei Facebook. Frankfurter Allgemeine Zeitung (FAZ), 19.

Assadollahi, R. (15. Mai 2018). Durch Künstliche Intelligenz geht die Arbeit nicht aus. Frankfurter Allgemeine Zeitung (FAZ), 18.

Beck, H. (10. März 2019). Auch Maschinen haben Vorurteile. Frankfurter Allgemeine Sonntagszeitung (FAS), 10.

Beschorner, T. (1. November 2018). Roboterethik: Eine Schlüsselfrage des 21. Jahrhunderts. Neue Züricher Zeitung (NZZ), 16.

Betschon, S. (24. September 2018). Die im Dunkeln pfeifen. Neue Züricher Zeitung (NZZ), 18.

Betschon, S. (13. Februar 2019a). Liebe Maschinen - Lasst uns doch Freunde sein! Neue Züricher Zeitung (NZZ), 17.

Betschon, S. (20. März 2019b). Künstliche Intelligenz als Idiot savant. Neue Züricher Zeitung (NZZ), 37.

Böge, F. (9. April 2019). Die künstliche Intelligenz vom Lande. Frankfurter Allgemeine Zeitung (FAZ), 3.

Budras, C. (22. Juli 2018). Künstliche Intelligenz muss fair sein. Frankfurter Allgemeine Sonntagszeitung (FAS), 21.

Budras, C. (14. April 2019). Unberechenbare Roboter. Frankfurter Allgemeine Sonntagszeitung (FAS), 20.

\footnotetext{
${ }^{24} \mathrm{Zu}$ diesen grundsätzlichen Fragen jetzt eindringlich auch Armin Grunwald (2019, S. 149 ff., 223 ff.).
} 
Clark, A. (8. November 2018). Leben in einer durchlässigen Realität. Neue Züricher Zeitung (NZZ), 21.

Datenschutzbeauftragter INFO. (17. Oktober 2017). Was bedeutet Privacy by Design/ Privacy by Default wirklich? https://www.datenschutzbeauftragter-info.de/wasbedeutet-privacy-by-design-privacy-by-default-wirklich/.

Die deutsche Bundesregierung. (18. Juli 2018). Eckpunkte der Bundesregierung für eine Strategie Künstliche Intelligenz [Parliamentary paper]. https://www.bmbf.de/ files/180718\%20Eckpunkte_KI-Strategie\%20final\%20Layout.pdf.

Digitalcourage e. V. (9 Mai 2014). Privacy by Default: Datenschutz darf keine Ausnahme bleiben. https://digitalcourage.de/blog/2014/privacy-default-datenschutz-darf-keineausnahme-bleiben.

Europäisches Parlament. (27 April 2016). Amtsblatt L119 - Der Europäischen Union [Parliamentary paper]. https://www.computerundrecht.de/OJ_L_2016_119_FULL_DE_ TXT.pdf.

European Commission. (18. Dezember 2018). Draft Ethics guidelines for trustworthy AI [Parliamentary paper]. https://ec.europa.eu/digital-single-market/en/news/draft-ethicsguidelines-trustworthy-ai.

European Commission. (8 April 2019). The ethics guidelines for trustworthy artificial intelligence (AI) [Parliamentary paper]. https://ec.europa.eu/futurium/en/ai-allianceconsultation/guidelines.

Feldle, J. (2018). Notstandsalgorithmen: Dilemmata im automatisierten Straßenverkehr. Baden-Baden: Nomos.

Frisch, M. (1962). Stücke I. (Santa Cruz/Nun singen sie wieder/Die Chinesische Mauer/Als der Krieg zu Ende war/Graf Öderland). Frankfurt a. M.: Suhrkamp.

Gabriel, M. (20. Mai 2018). Schlauer als jeder Mensch? Frankfurter Allgemeine Sonntagszeitung (FAS), 21.

Gigerenzer, G., Müller, K.-R., \& Wagner, G. (22. Juni 2018). Wie man Licht in die Black Box wirft. Frankfurter Allgemeine Zeitung (FAZ), 15.

Gillen, E. (10. Januar 2019a). Die Ethik-Falle. Frankfurter Allgemeine Zeitung (FAZ), 9.

Gillen, E. (24. April 2019b). Stets zu Diensten. Frankfurter Allgemeine Zeitung (FAZ), 13.

Glaser, P. (28. Dezember 2017). Die Verwandlung der Utopie in Waffentechnologie. Neue Züricher Zeitung (NZZ), 14.

Grunwald, A. (2019). Der unterlegene Mensch. Die Zukunft der Menschheit im Angesicht von Algorithmen, künstlicher Intelligenz und Robotern. München: riva.

Günther, J. P. (2016). Roboter und rechtliche Verantwortung: Eine Untersuchung der Benutzer- und Herstellerhaftung. München: Utz.

Günther, M. (13. Mai 2018). Wie wir Götter werden. Frankfurter Allgemeine Sonntagszeitung, 3 .

Hahn, U. (9. März 2019). Vernunft ist auch eine Herzenssache. Zum Verhältnis von Künstlicher Intelligenz und Literatur. Frankfurter Allgemeine Zeitung (FAZ), 16.

Henn, W. (25. Juni 2018). Wehe, die Computer sagen einmal ,ich“. Frankfurter Allgemeine Zeitung (FAZ), 15.

Hilgendorf, E., Hötitzsch, S., \& Lutz L. S. (Hrsg.). (2015). Rechtliche Aspekte automatisierter Fahrzeuge: Beiträge zur 2. Würzburger Tagung zum Technikrecht im Oktober 2014. Baden-Baden: Nomos. 
Hillmer, H.-J. (12 April 2019). Künstliche Intelligenz - Ethik-Leitlinien für die KI. https:// www.compliancedigital.de/ce/ethik-leitlinien-fuer-die-ki/detail.html.

Höffe, O. (7. Dezember 2017). Der Mensch ist kein Gott. Neue Züricher Zeitung (NZZ), 22. Ingold, F. P. (10. Oktober 2018). Die Muse küsst, wie und wen sie will. Neue Zürcher Zeitung (NZZ), 19.

Jonas, H. (1984). Das Prinzip Verantwortung: Versuch einer Ethik für die technologische Zivilisation. Frankfurt a. M.: Suhrkamp.

Kant, I. (1785). Grundlegung zur Metaphysik der Sitten. In W. Weischedel (Hrsg.), Werke in zehn Bänden (Bd. 6). Darmstadt: Wissenschaftliche Buchgesellschaft.

Kissinger, H. (16. Juni 2018). Warten auf die Philosophen: Mensch und Maschine. Die Welt, 2.

Klingler, W. (9. Januar 2018). Der neue radikale Maschinenkult. Der Digitalismus zelebriert sich als neue Heilslehre. Neue Züricher Zeitung (NZZ), 19.

Kreye, A. (24. November 2018). Berührungspunkte. Süddeutsche Zeitung, 13-15.

Lammert, N. (3. Dezember 2018). Die künstliche und die menschliche Intelligenz. Frankfurter Allgemeine Zeitung (FAZ), 8.

Lenzen, M. (2018a). Künstliche Intelligenz: Was sie kann \& was uns erwartet. München: Beck.

Lenzen, M. (20. November 2018b). Regeln für den Maschinenpark. Frankfurter Allgemeine Zeitung (FAZ), 13.

Lenzen, M. (23. März 2019). Träumen Androiden von Menschen? Frankfurter Allgemeine Zeitung (FAZ), 10.

Lobe, A. (9. Mai 2018). Big Data und Big History. Neue Züricher Zeitung (NZZ), 21.

Lobe, A. (3. Februar 2019). Gebaute Filterblasen. Algorithmische Systeme verstärken soziale Unterschiede. Frankfurter Allgemeine Sonntagszeitung (FAS), 39.

Lutz, L. S. (2015). Autonome Fahrzeuge als rechtliche Herausforderung. Neue Juristische Wochenschrift: NJW, 68(3), 119-124.

Maak, N. (14. Januar 2018). Nie mehr allein. Kein Land treibt die Robotisierung so voran wie Japan. Frankfurter Allgemeine Sonntagszeitung (FAS), 45.

Markschies, C. (23. Februar 2019). Warum soll man einem Computer vertrauen? Frankfurter Allgemeine Zeitung (FAZ), 11.

Mørch, H. H. (14. Januar 2018). Wie kommt der Geist in die Natur? Frankfurter Allgemeine Zeitung (FAZ), 65.

Müller, M. (7. Januar 2019). Die künstliche Intelligenz spricht Chinesisch. Neue Züricher Zeitung (NZZ), 34-35.

Nida-Rümelin, J. (7. Dezember 2016). Zwischen Euphorie und Apokalypse. Neue Züricher Zeitung (NZZ), 22.

Nosthoff, A.-V., \& Maschewski, F. (22. Februar 2019). Die App weiß, wann du stirbst. Neue Züricher Zeitung (NZZ), 19.

Picht, G. (1967). Der Begriff der Verantwortung. In K. Aland, \& W. Schneemelcher (Hrsg.), Kirche und Staat - Festschrift für Bischof D. D. Hermann Kunst zum 60. Geburtstag am 21. Januar 1967 (S. 189-223). Berlin: De Gruyter.

Rutkowski, M. (1. April 2019). Die komplett menschenleere Fabrik ist eine Fiktion. Frankfurter Allgemeine Zeitung, 22-23.

Ryffel, H. (1967). Verantwortung als sittliches Phänomen: Ein Grundzug moderner Praxis. Der Staat, 6(3), 275-292. 
Scheer, U. (19. März 2019). Sophia, warum siehst Du aus wie eine Frau? Frankfurter Allgemeine Zeitung (FAZ), 9.

Scherff, D. (26. Mai 2018). Roboter, fahr du voran. KI ersetzt den Fahrer im Auto. Frankfurter Allgemeine Sonntagszeitung (FAS), 23.

Schmidhuber, J. (12. Mai 2018). Künstliche Intelligenz ist eine Riesenchance für Deutschland. Frankfurter Allgemeine Zeitung (FAZ), 22.

Schnabel, U. (28. März 2018). Wenn die Maschinen immer klüger werden: Was macht uns künftig noch einzigartig? Die Zeit, 37-39.

Schwartmann, R. (25. Oktober 2018). Das Recht der Maschinen. Frankfurter Allgemeine Zeitung (FAZ), 8.

Spehr, M. (19. März 2019). Wie alles in die Cloud läuft. Frankfurter Allgemeine Zeitung (FAZ), T1.

Sprenger, R. K. (28. Januar 2019). Sie irrt sich nicht, das ist ihr Problem. Neue Züricher Zeitung (NZZ), 19.

Strasser, P. (30. Januar 2018). Das Ich verschwindet - Und jetzt? Neue Züricher Zeitung (NZZ), 15.

Strasser, P. (22. Januar 2019). Schwesterlichkeit - Spiritualität im 21. Jahrhundert. Neue Züricher Zeitung (NZZ), 15.

Surber, R. (24. April 2019). Auslagerung des Grundrechtsschutzes. Neue Züricher Zeitung (NZZ), 15.

Thielicke, H. (1957). Christliche Verantwortung im Atomzeitalter. Ethisch-politischer Traktat über einige Zeitfragen. Stuttgart: Evangelisches Verlagswerk.

von der Malsburg, C. (13. März 2019). Gesucht: Vorbilder für kluge Automaten. Frankfurter Allgemeine Zeitung, N1.

Weischedel, W. (1933). Das Wesen der Verantwortung. Ein Versuch. Frankfurt a. M.: Klostermann.

Widmer, H. (17. September 2018). Künstliche Intelligenz denkt nicht. Neue Züricher Zeitung (NZZ), 19.

Wikipedia - Die freie Enzyklopädie. (o. J.a). Datenschutz Grundverordnung. https:// de.wikipedia.org/wiki/Datenschutz-Grundverordnung.

Wikipedia - Die freie Enzyklopädie. (o. J.b). Industrie 4.0 - Umfassende Digitalisierung der industriellen Prozesse. https://de.wikipedia.org/wiki/Industrie_4.0.

Wikipedia - Die freie Enzyklopädie. (o. J.c). Privacy by design - Wikipedia. https:// en.wikipedia.org/wiki/Privacy_by_design.

Wikipedia - Die freie Enzyklopädie. (o. J.d). Transhumanismus - Wikipedia. https:// de.wikipedia.org/wiki/Transhumanismus.

Yogeshwar, R. (10. Januar 2019). Maschinen herrschen. Frankfurter Allgemeine Zeitung (FAZ), 9.

Ramge, T. (5. Februar 2018). Mensch fragt, Maschine antwortet. Wie künstliche Intelligenz Wirtschaft, Arbeit und unser Leben verändert. Aus Politik und Zeitgeschichte (APUZ) 68(6-8), 15-21.

Lankafel, P. (2015). Auf dem Weg zum Dr. Algorithmus?Potenziale von Big Data in der Medizin. Aus Politik und Zeitgeschichte (APUZ), 65(11), 27-32. 
Open Access Dieses Kapitel wird unter der Creative Commons Namensnennung 4.0 International Lizenz (http://creativecommons.org/licenses/by/4.0/deed.de) veröffentlicht, welche die Nutzung, Vervielfältigung, Bearbeitung, Verbreitung und Wiedergabe in jeglichem Medium und Format erlaubt, sofern Sie den/die ursprünglichen Autor(en) und die Quelle ordnungsgemäß nennen, einen Link zur Creative Commons Lizenz beifügen und angeben, ob Änderungen vorgenommen wurden.

Die in diesem Kapitel enthaltenen Bilder und sonstiges Drittmaterial unterliegen ebenfalls der genannten Creative Commons Lizenz, sofern sich aus der Abbildungslegende nichts anderes ergibt. Sofern das betreffende Material nicht unter der genannten Creative Commons Lizenz steht und die betreffende Handlung nicht nach gesetzlichen Vorschriften erlaubt ist, ist für die oben aufgeführten Weiterverwendungen des Materials die Einwilligung des jeweiligen Rechteinhabers einzuholen.

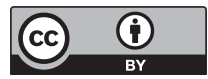

